Pacific

Journal of

Mathematics

ON THE EQUATIONS DEFINING

AFFINE ALGEBRAIC GROUPS

VLADIMIR L. POPOV

Volume $279 \quad$ No. 1-2

December 2015 


\title{
ON THE EQUATIONS DEFINING AFFINE ALGEBRAIC GROUPS
}

\author{
VLADIMIR L. POPOV \\ In memory of Robert Steinberg
}

\begin{abstract}
For the coordinate algebras of connected affine algebraic groups, we explore the problem of finding a presentation by generators and relations canonically determined by the group structure.
\end{abstract}

\section{Introduction}

Connected algebraic groups constitute a remarkable class of irreducible quasiprojective algebraic varieties. It contains the subclasses of abelian varieties and affine algebraic groups. These subclasses are basic: by Chevalley's theorem, every connected algebraic group $G$ has a unique connected normal affine algebraic subgroup $L$ such that $G / L$ is an abelian variety, whence the variety $G$ is an $L$-torsor over the abelian variety $G / L$. The varieties from these subclasses can be embedded in many ways as closed subvarieties in, respectively, projective and affine spaces. A natural question then arises as to whether there are distinguished embeddings and equations of their images, which are canonically determined by the group structure. For abelian varieties, this is the existence problem for canonically defined bases in linear systems and that of presenting homogeneous coordinate rings of ample invertible sheafs by generators and relations. These problems were explored and solved by D. Mumford [1966]. For affine algebraic groups, it is the existence problem of the canonically defined presentations of the coordinate algebras of such groups by generators and relations. We explore this problem in the present paper.

We fix as the base field an algebraically closed field $k$ of arbitrary characteristic. In this paper, as in [Borel 1991], "variety" means "algebraic variety" in the sense of Serre [1955, Subsection 34]; every variety is taken over $k$.

Let $G$ be a connected affine algebraic group and let $R_{u}(G)$ be its unipotent radical. In view of [Grothendieck 1958, Propositions 1, 2] and [Rosenlicht 1956, Theorem 10], the underlying variety of $G$ is isomorphic to the product of that of

Supported by grant РФФИ 14-01-00160.

MSC2010: primary 14L10; secondary 20G05.

Keywords: reductive algebraic group, Borel subgroup, weight, module, orbit. 
$G / R_{u}(G)$ and $R_{u}(G)$, and the latter is isomorphic to an affine space. Therefore, the problem under consideration is reduced to the case of reductive groups. Given this, henceforth $G$ stands for a connected reductive algebraic group.

The simplest case of $\mathrm{SL}_{2}$ is the guiding example. Take the polynomial $k$-algebra $k\left[x_{1}, x_{2}, x_{3}, x_{4}\right]$ in four variables $x_{i}$. The usual presentation of $k\left[\mathrm{SL}_{2}\right]$ is given by the surjective homomorphism

$$
\mu: k\left[x_{1}, x_{2}, x_{3}, x_{4}\right] \rightarrow k\left[\mathrm{SL}_{2}\right], \quad \mu\left(x_{i}\right)\left(\left[\begin{array}{ll}
a_{1} & a_{2} \\
a_{3} & a_{4}
\end{array}\right]\right)=a_{i},
$$

whose kernel is the ideal $\left(x_{1} x_{4}-x_{2} x_{3}-1\right)$. After rewriting, this presentation can be interpreted in terms of the group structure of $\mathrm{SL}_{2}$ as follows.

We have $k\left[x_{1}, x_{2}, x_{3}, x_{4}\right]=k\left[x_{1}, x_{3}\right] \otimes_{k} k\left[x_{2}, x_{4}\right]$ and the restriction of $\mu$ to the subalgebra $k\left[x_{1}, x_{3}\right]$ (respectively, $k\left[x_{2}, x_{4}\right]$ ) is an isomorphism with the subalgebra $\mathcal{S}^{+}$(respectively, $\mathcal{S}^{-}$) of $k\left[\mathrm{SL}_{2}\right]$ consisting of all regular functions invariant with respect to the subgroup $U^{+}$(respectively, $U^{-}$) of all unipotent upper (respectively, lower) triangular matrices acting by right translations. Hence (1) yields the following presentation of $k\left[\mathrm{SL}_{2}\right]$ by generators and relations:

$$
\begin{aligned}
k\left[\mathrm{SL}_{2}\right] & \cong\left(\mathcal{S}^{+} \otimes_{k} \mathcal{S}^{-}\right) / \mathcal{I}, \\
\mathcal{S}^{+} & =k\left[\mu\left(x_{1}\right), \mu\left(x_{3}\right)\right] \cong k\left[x_{1}, x_{3}\right], \\
\mathcal{S}^{-} & =k\left[\mu\left(x_{2}\right), \mu\left(x_{4}\right)\right] \cong k\left[x_{2}, x_{4}\right], \\
\mathcal{I} & =\left(\mu\left(x_{1}\right) \otimes \mu\left(x_{4}\right)-\mu\left(x_{2}\right) \otimes \mu\left(x_{3}\right)-1\right) .
\end{aligned}
$$

The subgroups $U^{+}, U^{-}$are opposite maximal unipotent subgroups of $\mathrm{SL}_{2}$. The subalgebras $\mathcal{S}^{+}, \mathcal{S}^{-}$are stable with respect to $\mathrm{SL}_{2}$ acting by left translations, and $f:=\mu\left(x_{1}\right) \otimes \mu\left(x_{4}\right)-\mu\left(x_{2}\right) \otimes \mu\left(x_{3}\right)-1$ is the unique element of $\left(\mathcal{S}^{+} \otimes_{k} \mathcal{S}^{-}\right)^{\mathrm{SL}_{2}}$ determined by the conditions $f(e, e)=1, k[f]=\left(\mathcal{S}^{+} \otimes_{k} \mathcal{S}^{-}\right)^{\mathrm{SL}_{2}}$.

We show that there is an analogue of (2) for every connected reductive algebraic group $G$. Namely, we endow $k[G]$ with the $G$-module structure determined by left translations and fix in $G$ a pair of opposite Borel subgroups $B^{+}$and $B^{-}$. Let $U^{ \pm}$ be the unipotent radical of $B^{ \pm}$. Consider the $G$-stable subalgebras

$$
\begin{aligned}
& \mathcal{S}^{+}:=\left\{f \in k[G] \mid f(g u)=f(g) \text { for all } g \in G, u \in U^{+}\right\}, \\
& \mathcal{S}^{-}:=\left\{f \in k[G] \mid f(g u)=f(g) \text { for all } g \in G, u \in U^{-}\right\}
\end{aligned}
$$

of $k[G]$ and the natural multiplication homomorphism of $k$-algebras

$$
\mu: \mathcal{S}^{+} \otimes_{k} \mathcal{S}^{-} \rightarrow k[G], \quad f_{1} \otimes f_{2} \mapsto f_{1} f_{2} .
$$

For $k=\mathbb{C}$, the following were put forward in [Flath and Towber 1992]:

Conjectures (D. E. Flath and J. Towber [1992]).

(S) The homomorphism $\mu$ is surjective.

(K) The ideal ker $\mu$ in $\mathcal{S}^{+} \otimes_{k} \mathcal{S}^{-}$is generated by $(\operatorname{ker} \mu)^{G}$. 
If these conjectures are true, then the problem under consideration is reduced to the following:

(a) Find the canonically defined generators of the $k$-algebra $(\operatorname{ker} \mu)^{G}$.

(b) Find the canonically defined presentations of $\mathcal{S}^{ \pm}$by generators and relations.

In [Flath and Towber 1992], Conjectures (S) and (K) were proved for $k=\mathbb{C}$ and $G=\mathrm{SL}_{n}, \mathrm{GL}_{n}, \mathrm{SO}_{n}, \mathrm{Sp}_{n}$ by means of lengthy direct computations of some Laplace decompositions, minors, and algebraic identities between them. In Theorems 3 and 9 below, we prove Conjectures $(\mathrm{S})$ and $(\mathrm{K})$ in full generality, with no restrictions on $k$ and $G$.

In Theorems 11 and 20 below, we describe $\operatorname{ker} \mu$ as a vector space over $k$. In Theorem 21, we solve the above part (a) of the problem, finding the canonically defined generators of the $k$-algebra $(\operatorname{ker} \mu)^{G}$. We call them $\mathrm{SL}_{2}$-type relations of the sought-for canonical presentation of $k[G]$ because for $G=\mathrm{SL}_{2}$, the element $\mu\left(x_{1}\right) \otimes \mu\left(x_{4}\right)-\mu\left(x_{2}\right) \otimes \mu\left(x_{3}\right)-1$ is just such a generator of $\mathcal{I}$ (see (2)). All of them are inhomogeneous of degree 2. If $G$ is semisimple, they are indexed by the elements of the Hilbert basis $\mathscr{H}$ of the monoid of dominant weights of $G$. Note that the cardinality $|\mathscr{H}|$ of $\mathscr{H}$ is at least rank $G$ with equality for simply connected $G$, but in the general case it may be much bigger. For instance, if $G=\mathrm{PGL}_{r}$, then $|\mathscr{H}| \geqslant p(r)+\varphi(r)-1$, where $p$ and $\varphi$ are, respectively, the classical partition function and the Euler function (see [Popov 2011, Example 3.15]). Note that the problem of determining a full set of generators of the ideal ker $\mu$ was formulated in [Flath 1994, Section 4] and, for $k=\mathbb{C}, G=\mathrm{SL}_{n}, \mathrm{GL}_{n}, \mathrm{SO}_{n}, \mathrm{Sp}_{n}$, solved in [Flath and Towber 1992] by lengthy direct computations.

For a semisimple group $G$ whose monoid of dominant weights is freely generated (i.e., with $|\mathscr{H}|=\operatorname{rank} G$ ), a solution to the above part (b) of the problem in characteristic 0 was obtained (but not published) by B. Kostant; his proof appeared in [Lancaster and Towber 1979, Theorem 1.1]. In arbitrary characteristic, such a solution is given by Theorems 1, 2, 22 below, which are heavily based on the main results of [Ramanan and Ramanathan 1985] and [Kempf and Ramanathan 1987]. All relations in this case are homogeneous of degree 2. We call them Plücker-type relations of the sought-for canonical presentation of $k[G]$ because the $k$-algebra $\mathcal{S}^{ \pm}$ for $G=\mathrm{SL}_{n}$ is the coordinate algebra of the affine multicone over the flag variety, and if char $k=0$, these relations are generated by the classical Plücker-type relations, obtained by Hodge $[1942 ; 1943]$, that determine this multicone (see Section 6). The set of these relations is a union of finite-dimensional vector spaces canonically determined by the group structure of $G$; these spaces are indexed by the elements of $\mathscr{H} \times \mathscr{H}$ and different spaces have zero intersection (see Theorem 22). Thus in this case, we obtain a canonical presentation of $k[G]$, in which all relations are quadratic and divided into two families: homogeneous relations of Plücker type and 
inhomogeneous relations of $\mathrm{SL}_{2}$-type. As a parallel, we recall that any abelian variety is canonically presented as an intersection of quadrics in a projective space given by the Riemann equations; see [Kempf 1989] and [Lange and Birkenhake 1992].

For an arbitrary reductive group $G$, let $\tau: \widehat{G} \rightarrow G$ be the universal covering. Then $\widehat{G}=Z \times C$, where $Z$ is a torus, $C$ is a simply connected semisimple group, $G=\widetilde{G} / \operatorname{ker} \tau$, and $\operatorname{ker} \tau$ is a finite central subgroup. The algebra $\mathcal{S}^{ \pm}$for $\widehat{G}$ is then the tensor product of $k[Z]$ and the algebra $\mathcal{S}^{ \pm}$for $C$. Since the presentation of $k[Z]$ is clear, and that of $\mathcal{S}^{ \pm}$for $C$ are given by Theorems 1,2 , and 22, the above part (b) of the problem is reduced to finding a presentation for the invariant algebra of the finite abelian group $\operatorname{ker} \tau$.

As an illustration, in Section 6 we consider the example of $G=\mathrm{SL}_{n}$, char $k=0$, and describe explicitly how the ingredients of our construction and the canonical presentation of $k[G]$ look in this case.

The preprints [Popov 1995; 2000] of these results in characteristic 0 have been disseminated long ago. The validity of the results in arbitrary characteristic was announced in [Popov 2000]. The author is happy to finally present the complete proofs in the volume dedicated to the memory of Robert Steinberg who made a great contribution to the theory of algebraic groups.

Notation and conventions. Below we use freely the standard notation and conventions of [Borel 1991; Jantzen 1987; Popov and Vinberg 1994; Shafarevich 2013]. In particular, the algebra of functions regular on a variety $X$ is denoted by $k[X]$, the field of rational functions on an irreducible $X$ is denoted by $k(X)$, and the local ring of $X$ at a point $x$ is denoted by $\mathcal{O}_{x, X}$. For a morphism $\varphi: X \rightarrow Y$ of varieties, $\varphi^{*}: k[Y] \rightarrow k[X]$ denotes its comorphism.

All topological terms refer to the Zariski topology; the closure of $Z$ in $X$ is denoted by $\bar{Z}$ (each time it is clear from the context what is $X$ ).

The fixed point set of an action of a group $P$ on a set $S$ is denoted by $S^{P}$. Every action $\alpha: H \times X \rightarrow X$ of an algebraic group $H$ on a variety $X$ is always assumed to be regular (the latter means that $\alpha$ is a morphism). For every $h \in H, x \in X$, we write $g \cdot x$ in place of $\alpha(g, x)$. The $H$-orbit and the $H$-stabilizer of $x$ are denoted respectively by $H \cdot x$ and $H_{x}$. Every homomorphism of algebraic groups is assumed to be algebraic.

The additively written group of characters (i.e., homomorphisms to the multiplicative group of $k$ ) of an algebraic group $H$ is denoted by $\mathrm{X}(H)$. The value of a character $\lambda \in \mathrm{X}(H)$ at an element $h \in H$ is denoted by $h^{\lambda}$. Given a $k H$-module $M$, its weight space with weight $\lambda \in X(H)$ is denoted by $M_{\lambda}$.

We fix in $G$ the maximal torus

$$
T:=B^{+} \cap B^{-}
$$

and identify $\mathrm{X}\left(B^{ \pm}\right)$with $\mathrm{X}(T)$ by means of the restriction isomorphisms $\mathrm{X}\left(B^{ \pm}\right) \rightarrow$ $\mathrm{X}(T),\left.\lambda \mapsto \lambda\right|_{T}$. 
By $\mathrm{X}(T)_{+}$we denote the monoid of dominant weights of $T$ determined by $B^{+}$. Below the highest weight of every simple $G$-module is assumed to be the highest weight with respect to $T$ and $B^{+}$.

We denote by $w_{0}$ the longest element of the Weyl group of $T$ and fix in the normalizer of $T$ a representative $\dot{w}_{0}$ of $w_{0}$. We then have $\dot{w}_{0} B^{ \pm} \dot{w}_{0}^{-1}=B^{\mp}$ and $\dot{w}_{0} U^{ \pm} \dot{w}_{0}^{-1}=U^{\mp}$. For every $\lambda \in \mathrm{X}(T)_{+}$, we put $\lambda^{*}:=-w_{0}(\lambda) \in \mathrm{X}(T)_{+}$.

The set of all nonnegative rational numbers is denoted by $\mathbb{Q} \geqslant 0$ and we put $\mathbb{N}:=\mathbb{Z} \cap \mathbb{Q} \geqslant 0$.

If $m \in \mathbb{Z}, m>0$, we put $[m]:=\{a \in \mathbb{Z} \mid 1 \leqslant a \leqslant m\}$.

For $d \in \mathbb{N}$, we denote by $[m]_{d}$ the set of all increasing sequences of $d$ elements of $[m]$ (if $d \notin[m]$, then $[m]_{d}=\varnothing$ ).

\section{Proof of Conjecture (S)}

For every $\lambda \in \mathrm{X}(T)$, the spaces

$$
\begin{aligned}
& \mathcal{S}^{+}(\lambda):=\left\{f \in \mathcal{S}^{+} \mid f(g t)=t^{\lambda} f(g) \text { for all } g \in G, t \in T\right\}, \\
& \mathcal{S}^{-}(\lambda):=\left\{f \in \mathcal{S}^{-} \mid f(g t)=t^{w_{0}(\lambda)} f(g) \text { for all } g \in G, t \in T\right\}
\end{aligned}
$$

are the finite-dimensional (see, e.g., [Jantzen 1987, I.5.12.c)]) $G$-submodules of the $G$-modules $\mathcal{S}^{+}$and $\mathcal{S}^{-}$respectively. Since $\mathcal{S}^{-}(\lambda)$ is the right translation of $\mathcal{S}^{+}(\lambda)$ by $\dot{w}_{0}$, these $G$-submodules are isomorphic. In the notation of [Jantzen 1987, II.2.2], we have

$$
\mathcal{S}^{-}(\lambda)=H^{0}\left(\lambda^{*}\right),
$$

so by (6) and [Jantzen 1987, II.2.6, 2.2, 2.3], the following properties hold:

(i) $\mathcal{S}^{ \pm}(\lambda) \neq 0 \Longleftrightarrow \lambda \in \mathrm{X}(T)_{+}$.

(ii) $\operatorname{soc}_{G} \mathcal{S}^{ \pm}(\lambda)$ is a simple $G$-module with the highest weight $\lambda^{*}$.

If char $k=0$, then the $G$-module $\mathcal{S}^{+}(\lambda)$ is semisimple and hence $\mathcal{S}^{+}(\lambda)=$ $\operatorname{soc}_{G} \mathcal{S}^{+}(\lambda)$ by (7)(ii). If char $k>0$, then, in general, this equality does not hold. From (3), (5), and (7)(i) we infer that

$$
\begin{aligned}
& \mathcal{S}^{+}=\bigoplus_{\lambda \in \mathrm{X}(T)_{+}} \mathcal{S}^{+}(\lambda), \quad \mathcal{S}^{+}(\lambda) \mathcal{S}^{+}(\mu) \subseteq \mathcal{S}^{+}(\lambda+\mu), \\
& \mathcal{S}^{-}=\bigoplus_{\lambda \in \mathrm{X}(T)_{+}} \mathcal{S}^{-}(\lambda), \quad \mathcal{S}^{-}(\lambda) \mathcal{S}^{-}(\mu) \subseteq \mathcal{S}^{-}(\lambda+\mu)
\end{aligned}
$$

i.e., the decompositions (8) are the $\mathrm{X}(T)_{+}$-gradings of the algebras $\mathcal{S}^{+}$and $\mathcal{S}^{-}$. They are obtained from each other by the right translation by $\dot{w}_{0}$.

Theorem 1. The linear span of $\mathcal{S}^{ \pm}(\lambda) \mathcal{S}^{ \pm}(\mu)$ over $k$ is $\mathcal{S}^{ \pm}(\lambda+\mu)$. 
Proof. This statement is the main result of [Ramanan and Ramanathan 1985]. Note that the difficulty lies in the case of positive characteristic: since $\mathcal{S}^{ \pm}$is an integral domain, if char $k=0$, then the claim immediately follows from (7)(i) and the inclusions in (8) because then $\mathcal{S}^{ \pm}(\lambda+\mu)$ is a simple $G$-module.

Theorem 2. (i) If $\varphi$ is a generating set of the semigroup $\mathrm{X}(T)_{+}$, then the $k$-algebra $\mathcal{S}^{ \pm}$is generated by the subspace $\bigoplus_{\lambda \in \mathscr{G}} \mathcal{S}^{ \pm}(\lambda)$.

(ii) The $k$-algebras $S^{+}$and $\mathcal{S}^{-}$are finitely generated.

Proof. Part (i) follows from (8) and Theorem 1. Being the intersection of the lattice $\mathrm{X}(T)$ with a convex cone in $\mathrm{X}(T) \otimes_{\mathbb{Z}} \mathbb{Q}$ generated by finitely many vectors, the semigroup $\mathrm{X}(T)_{+}$is finitely generated. This, (i), and the inequality $\operatorname{dim}_{k} \mathcal{S}^{ \pm}(\lambda)<\infty$ imply (ii).

Now we are ready to turn to the proof of Conjecture (S).

Theorem 3. The homomorphism $\mu$ is surjective.

Our proof of Theorem 3 is based on two general results. The first is the following well-known surjectivity criterion:

Lemma 4. The following properties of a morphism $\varphi: X \rightarrow Y$ of affine algebraic varieties are equivalent:

(a) $\varphi$ is a closed embedding.

(b) $\varphi^{*}: k[Y] \rightarrow k[X]$ is surjective.

Proof. See, e.g., [Steinberg 1974, Section 1.5].

The second is the closedness criterion for orbits of connected solvable affine algebraic groups that generalizes Rosenlicht's classical theorem [1961, Theorem 2] on the closedness of orbits of unipotent groups.

Theorem 5. Let a connected solvable affine algebraic group $S$ act on an affine algebraic variety $Z$. Let $x$ be a point of $Z$. Consider the orbit morphism $\tau: S \rightarrow Z$, $s \mapsto s \cdot z$. Then the following properties are equivalent:

(a) The orbit $S \cdot z$ is closed in $Z$.

(b) The semigroup $\left\{\lambda \in \mathrm{X}(S) \mid\right.$ the function $S \rightarrow k, s \mapsto s^{\lambda}$, lies in $\left.\tau^{*}(k[Z])\right\}$ is a group.

Proof. This is proved in [Popov 1989, Theorem 4]

Remark 6. Since $\mathrm{X}(S)$ in Theorem 5 is a finitely generated free abelian group, it can be naturally regarded as a lattice in $\mathrm{X}(S) \otimes_{\mathbb{Z}} \mathbb{Q}$. Hence the following general criterion is applicable for verifying condition (b).

Let $M$ be a nonempty subset of a finite-dimensional vector space $V$ over $\mathbb{Q}$. Let $\mathbb{Q} \geqslant 0 M$, conv $M$, and $\mathbb{Q} M$ be, respectively, the convex cone generated by $M$, the 
convex hull of $M$, and the linear span of $M$ in $V$. Then the following properties are equivalent (see [Popov 1989, p. 386]):

(i) 0 is an interior point of conv $M$.

(ii) $\mathbb{Q} \geqslant 0 M=\mathbb{Q} M$.

If $M$ is a subsemigroup of $V$, then (i) and (ii) are equivalent to

(iii) $M$ is a group.

Proof of Theorem 3. 1. We consider the action of $G$ on its underlying algebraic variety by left translations. By Theorem 2, there is an irreducible affine algebraic variety $X$ endowed with an action of $G$ and a $G$-equivariant dominant morphism

$$
\alpha: G \rightarrow X \text { such that } \alpha^{*} \text { is an isomorphism } k[X] \stackrel{\cong}{\longrightarrow} \mathcal{S}^{+} .
$$

Let $x:=\alpha(e)$. Since $\alpha$ is $G$-equivariant, we have

$$
\alpha(g)=g \cdot x \text { for every } g \in G,
$$

and since $\alpha$ is dominant, the orbit $G \cdot x$ is open and dense in $X$. Consider the canonical projection $\pi: G \rightarrow G / U^{+}$. It is the geometric quotient for the action of $U^{+}$on $G$ by right translations. Therefore, (3) yields the isomorphism

$$
\pi^{*}: k\left[G / U^{+}\right] \stackrel{\cong}{\longrightarrow} \mathcal{S}^{+},
$$

and, since $\alpha$ is constant on the fibers $\pi$, there exists a $G$-equivariant morphism $\iota: G / U^{+} \rightarrow X$ such that

$$
\alpha=\iota \circ \pi
$$

From (12) we infer that the image of $\iota$ is $G \cdot x$. Since the group $U^{+}$is unipotent, the algebraic variety $G / U^{+}$is quasiaffine (see [Rosenlicht 1961, Theorem 3]). Therefore, $k\left(G / U^{+}\right)$is the field of fractions of $k\left[G / U^{+}\right]$. On the other hand, $k(X)$ is the field of fractions of $k[X]$ inasmuch as $X$ is affine. Using that (12) and isomorphisms (9), (11) yield the isomorphism $\iota^{*}: k[X] \cong k\left[G / U^{+}\right]$, we conclude that $\iota$ is a birational isomorphism. Therefore, for a point $z$ in general position in $G \cdot x$, the fiber $\iota^{-1}(z)$ is a single point. Being $G$-equivariant, $\iota$ is then injective. Finally, since $G$ is smooth, $k[G]$ is integrally closed; therefore, $\mathcal{S}^{ \pm}$is integrally closed as well in view of (3) (see, e.g., [Popov and Vinberg 1994, Theorem 3.16]). Thus $X$ is normal, and hence by Zariski's Main Theorem, $\iota: G / U^{+} \rightarrow G \cdot x$ is an isomorphism. Using that $\pi$ is separable (see, e.g., [Borel 1991, II.6.5]), from this we infer that the following properties hold:

(i $\left.i_{1}\right) G_{x}=U^{+}$.

(iii $) G \rightarrow G \cdot x, g \mapsto \alpha(g)=g \cdot x$, is a separable morphism. 
2. Let $y:=\dot{w}_{0} \cdot x$. Consider the $G$-equivariant morphism

$$
\beta: G \rightarrow X, \quad g \mapsto g \cdot y .
$$

From (10), (13), ( $\left.i_{1}\right)$, and (ii 1$)$, we infer that the following properties hold:

(i2) $G_{y}=U^{-}$.

(ii 2$) ~ G \rightarrow G \cdot y, g \mapsto \beta(g)=g \cdot y$, is a separable morphism.

(iii 2$) \beta^{*}$ is an isomorphism $k[X] \stackrel{\cong}{\longrightarrow} \mathcal{S}^{-}$.

3. Now consider the $G$-equivariant morphism

$$
\gamma:=\alpha \times \beta: G \rightarrow X \times X, \quad g \mapsto g \cdot z, \text { where } z:=(x, y) .
$$

From $(14),\left(i_{1}\right)$, and $\left(i_{2}\right)$, we obtain

$$
G_{z}=G_{x} \cap G_{y}=U^{+} \cap U^{-}=\{e\},
$$

and hence $\gamma$ is injective. We claim that $\gamma$ is a closed embedding, i.e.,

(a) $G \rightarrow G \cdot z, g \mapsto g \cdot z$, is an isomorphism;

(b) $G \cdot z$ is closed in $X \times X$.

If this claim is proved, then the proof of Theorem 3 is completed as follows. Consider the isomorphism

$$
k[X] \otimes_{k} k[X] \rightarrow k[X \times X], \quad f \otimes h \mapsto f h .
$$

Then (4), (9), (iii 2$),(14),(16)$ imply that $\mu$ is the composition of the homomorphisms

$$
\mathcal{S}^{+} \otimes_{k} \mathcal{S}^{-} \underset{\cong}{\cong} k[X] \otimes_{k} k[X] \stackrel{(16)}{\cong} k[X \times X] \stackrel{\gamma^{*}}{\longrightarrow} k[G] .
$$

Hence the surjectivity of $\mu$ is equivalent to the surjectivity of $\gamma^{*}$. By Lemma 4, the latter is equivalent to the property that $\gamma$ is a closed embedding, i.e., that properties (a) and (b) hold.

Thus the proof of Theorem 3 is reduced to proving properties (a) and (b).

4. First, we shall prove property (a). Since $\gamma$ is injective, this is reduced to proving the separability of $\gamma$. In turn, in view of (14), the latter is reduced to proving that $\operatorname{ker} d_{e} \gamma$ is contained in Lie $G_{z}$, i.e., that $\operatorname{ker} d_{e} \gamma=\{0\}$ because of (15) (see [Borel 1991, II.6.7]). Using [loc. cit.], from (10), (13), (i $\left.i_{1}\right),\left(\mathrm{ii}_{1}\right),\left(\mathrm{i}_{2}\right),\left(\mathrm{ii}_{2}\right)$ we infer that $\operatorname{ker} d_{e} \alpha \subseteq$ Lie $U^{+}, \operatorname{ker} d_{e} \beta \subseteq$ Lie $U^{-}$. In view of (14), we then have $\operatorname{ker} d_{e} \gamma=\operatorname{ker} d_{e} \alpha \cap \operatorname{ker} d_{e} \beta \subseteq \operatorname{Lie} U^{+} \cap \operatorname{Lie} U^{-}=\{0\}$. This proves property (a).

5. Now we shall prove property (b). Actually, we shall prove the stronger property that the orbit $B^{+} \cdot z$ is closed in $X \times X$ : since the algebraic variety $G / B^{+}$is complete, this stronger property implies property (b) (see [Steinberg 1974, Section 2.13, Lemma 2]). Using that $B^{+}$is connected solvable, to this end we shall apply Theorem 5. 
Namely, consider the morphism $\tau: B^{+} \rightarrow X \times X, b \mapsto b \cdot z$ and the following subsemigroup $M$ in $\mathrm{X}\left(B^{+}\right)$:

$$
M:=\left\{\lambda \in \mathrm{X}\left(B^{+}\right) \mid \text {the function } B^{+} \rightarrow k, b \mapsto b^{\lambda} \text { lies in } \tau^{*}(k[X \times X])\right\} .
$$

We identify $\mathrm{X}\left(B^{+}\right)$with the lattice in $L:=\mathrm{X}\left(B^{+}\right) \otimes_{\mathbb{Z}} \mathbb{Q}$. In view of Theorem 5 and Remark 6, the orbit $B^{+} \cdot z$ is closed if and only if

$$
\mathbb{Q} \geqslant 0 M=\mathbb{Q} M \text {. }
$$

Given this, the problem is reduced to proving that property (18) holds. This is done below.

6. Since $\tau=\left.\gamma\right|_{B^{+}}$, the algebra $\tau^{*}(k[X \times X])$ is the image of the homomorphism $\gamma^{*}(k[X \times X]) \rightarrow k\left[B^{+}\right],\left.f \mapsto f\right|_{B^{+}}$. From (17) we see that $\gamma^{*}(k[X \times X])$ contains $\mathcal{S}^{+}$and $\mathcal{S}^{-}$. Hence the restrictions of $\mathcal{S}^{+}$and $\mathcal{S}^{-}$to $B^{+}$lie in $\tau^{*}(k[X \times X])$. We shall exhibit some characters of $B^{+}$lying in these restrictions.

First consider the restriction of $\mathcal{S}^{+}(\lambda)$ to $B^{+}$for $\lambda \in \mathrm{X}(T)_{+}$. Note that $\mathcal{S}^{+}(\lambda)$ contains a function $f$ such that $f(e) \neq 0$. Indeed, in view of (7)(i) and Borel's fixed point theorem, $\mathcal{S}^{+}(\lambda)$ contains a $B^{-}$-stable line $\ell$. The group $B^{-}$acts on $\ell$ by means of a character $v \in \mathrm{X}\left(B^{-}\right)$. Take a nonzero function $f \in \ell$. For every $b \in B^{-}, u \in U^{+}$, we then have $f\left(b^{-1} u\right)=b^{v} f(u) \stackrel{(3)}{=} b^{v} f(e)$, whence $f(e) \neq 0$ because $B^{-} U^{+}$is dense in $G$. This proves the existence of $f$. Multiplying $f$ by $1 / f(e)$, we may assume that $f(e)=1$. Then for every $b \in B^{+}$, we deduce from (3), (5) that $f(b)=$ $b^{\lambda} f(e)=b^{\lambda}$, i.e., $\left.f\right|_{B^{+}}$is the character $B^{+} \rightarrow k, b \mapsto b^{\lambda}$. This proves the inclusion

$$
\mathrm{X}\left(B^{+}\right)_{+} \subseteq M \text {. }
$$

Now consider the restriction of $\mathcal{S}^{-}(\lambda)$ to $B^{+}$for $\lambda \in \mathrm{X}(T)_{+}$. In view of (7)(ii), there is a $B^{+}$-stable line $\ell$ in $\mathcal{S}^{-}(\lambda)$, on which $B^{+}$acts by the character $\lambda^{*} \in \mathrm{X}\left(B^{+}\right)$. Take a nonzero function $f \in \ell$. We may assume that $f(e)=1$ : this is proved as above with $v=\lambda$, replacing $B^{-}$by $B^{+}$, and $U^{+}$by $U^{-}$. For every $b \in B^{+}$, we then have $f\left(b^{-1}\right)=b^{\lambda^{*}}$, i.e., $\left.f\right|_{B^{+}}$is the character $B^{+} \rightarrow k, b \mapsto b^{-\lambda^{*}}=b^{w_{0}(\lambda)}$. This proves the inclusion

$$
-\mathrm{X}\left(B^{+}\right)_{+} \subseteq M \text {. }
$$

Since $\mathbb{Q}_{\geqslant 0}\left(\mathrm{X}\left(B^{+}\right)_{+}\right)-\mathbb{Q}_{\geqslant 0}\left(\mathrm{X}\left(B^{+}\right)_{+}\right)=L$, the inclusions (19), (20) imply the equality $\mathbb{Q}_{\geqslant 0} M=L$, whence a fortiori the equality (18) holds. This completes the proof of Theorem 3 .

\section{Proof of Conjecture (K)}

We now intend to describe the ideal $\operatorname{ker} \mu$ in $\mathcal{S}^{+} \otimes_{k} \mathcal{S}^{-}$. This is done in Sections 3 and 4 in several steps: first in Theorem 9 we prove that $\operatorname{ker} \mu$ is generated by 
$(\operatorname{ker} \mu)^{G}$, then in Theorem 11 we describe $\operatorname{ker} \mu$ as a vector space, and finally in Theorem 21 we find a standard finite generating set of ker $\mu$.

The first step is based on the following general statement:

Theorem 7. Let $Z$ be an affine algebraic variety endowed with an action of a reductive algebraic group $H$. Let $a \in Z$ be a point such that the orbit morphism

$$
\varphi: H \rightarrow Z, \quad h \mapsto h \cdot a,
$$

is a closed embedding. Then the ideal $\operatorname{ker} \varphi^{*}$ in $k[Z]$ is generated by $\left(\operatorname{ker} \varphi^{*}\right)^{H}$.

For the proof of Theorem 7, we need the following:

Lemma 8. Let $\psi: Y \rightarrow Z$ be a morphism of irreducible affine algebraic varieties and let $z \in \psi(Y)$ be a smooth point of $Z$. Assume that for each point $y \in \psi^{-1}(z)$, the following hold:

(i) $y$ is a smooth point of $Y$.

(ii) The differential $d_{y} \psi$ is surjective.

Then the ideal $\left\{f \in k[Y]|f|_{\psi^{-1}(z)}=0\right\}$ of $k[Y]$ is generated by $\psi^{*}(\mathfrak{m})$, where $\mathfrak{m}:=\{h \in k[Z] \mid h(z)=0\}$.

Proof. Given a nonzero function $f \in k[Y]$, below we denote by $Y_{f}$ the principal open subset $\{y \in Y \mid f(y) \neq 0\}$ of $Y$; it is affine and $k\left[Y_{f}\right]=k[Y]_{f}$.

1. Let $s_{1}, \ldots, s_{d}$ be a system of generators of the ideal $\mathfrak{m}$ of $k[Z]$. Put $t_{i}:=\psi^{*}\left(s_{i}\right)$. Then we have

$$
\left\{y \in Y \mid t_{1}(y)=\cdots=t_{d}(y)=0\right\}=\psi^{-1}(z) .
$$

We claim that, for every point $a \in Y$, there is a function $h_{a} \in k[Y]$ such that the principal open subset $U=Y_{h_{a}}$ is a neighborhood of $a$ and

$$
I_{U}:=\left\{f \in k[U]|f|_{\psi^{-1}(z) \cap U}=0\right\}
$$

is the ideal of $k[U]$ generated by $\left.t_{1}\right|_{U}, \ldots,\left.t_{d}\right|_{U}$.

Proving this, we consider two cases.

First, consider the case where $a \notin \psi^{-1}(z)$. Then any principal open neighborhood of $a$ not intersecting $\psi^{-1}(z)$ may be taken as $U$ because in this case $I_{U}=k[U]$ and, in view of (21) and Hilbert's Nullstellensatz, $k[U]=\left.k[U] t_{1}\right|_{U}+\cdots+\left.k[U] t_{d}\right|_{U}$.

Second, consider the case where $a \in \psi^{-1}(z)$. Let $n=\operatorname{dim} Y, m=\operatorname{dim} Z$. Since $a$ and $z$ are the smooth points, the assumption (ii) yields the equality

$$
\operatorname{dim} \operatorname{ker} d_{a} \psi=n-m .
$$

The functions $s_{1}, \ldots, s_{d}$ generate the maximal ideal of $\mathcal{O}_{z, Z}$. Therefore, renumbering them if necessary, we may (and shall) assume that $s_{1}, \ldots, s_{m}$ is a system of local parameters of $Z$ at $z$, i.e., $\bigcap_{i=1}^{m} \operatorname{ker} d_{z} s_{i}=\{0\}$. Since $d_{a} t_{i}=d_{a} \psi \circ d_{z} s_{i}$, 
we then infer from (ii) that $\bigcap_{i=1}^{m} \operatorname{ker} d_{a} t_{i}=\operatorname{ker} d_{a} \psi$. In view of (22), the latter equality implies the existence of functions $f_{1}, \ldots, f_{n-m} \in \mathcal{O}_{a, Y}$ such that $t_{1}, \ldots, t_{m}, f_{1}, \ldots, f_{n-m}$ is a system of local parameters of $Y$ at $a$. Let

$$
F:=\left\{y \in Y \mid t_{1}(y)=\cdots=t_{m}(y)=0\right\} .
$$

By [Shafarevich 2013, Chapter II, Section 3.2, Theorem 2.13], there is a principal open neighborhood $U$ of $a$ such that $F \cap U$ is an irreducible smooth $(n-m)$-dimensional closed subvariety of $U$ whose ideal in $k[U]$ is generated by $\left.t_{1}\right|_{U}, \ldots,\left.t_{m}\right|_{U}$. On the other hand, (21) and (23) yield $\psi^{-1}(z) \subseteq F$ and, by the fiber dimension theorem, every irreducible component of $\psi^{-1}(z)$ has dimension $\geqslant n-m$. Hence $U \cap F=\psi^{-1}(z) \cap U$. This and (21) prove the claim. 2. Using this claim, the proof of Lemma 8 is completed as follows. Since $Y=$ $\bigcup_{a \in Y} Y_{h_{a}}$ and $Y$ is quasicompact, there exists a finite set of points $a_{1}, \ldots, a_{r} \in Y$ such that

$$
Y=\bigcup_{i=1}^{r} Y_{h_{i}}, \quad \text { where } h_{i}:=h_{a_{i}} .
$$

Now, let $f \in k[Y]$ be a function such that $\left.f\right|_{\psi^{-1}(z)}=0$. Then, in view of the definition of $h_{a}$, for every $i=1, \ldots, r$, we have

$$
f h_{i}^{b_{i}}=c_{i, 1} t_{1}+\cdots+c_{i, d} t_{d} \quad \text { for some } c_{i, j} \in k[Y] \text { and } b_{i} \in \mathbb{N} .
$$

From (24) and Hilbert's Nullstellensatz, we infer that there are functions $q_{1}, \ldots, q_{r} \in$ $k[Y]$ such that

$$
1=q_{1} h_{1}^{b_{1}}+\cdots+q_{s} h_{s}^{b_{r}} .
$$

From (25) and (26), we then deduce that

$$
f=\left(\sum_{i=1}^{r} q_{i} c_{i, 1}\right) t_{1}+\cdots+\left(\sum_{i=1}^{r} q_{i} c_{i, d}\right) t_{d} \in k[Y] t_{1}+\cdots+k[Y] t_{d} .
$$

Proof of Theorem 7. There is a closed equivariant embedding of $Z$ in an affine space on which $H$ operates linearly (see [Rosenlicht 1961, Lemma 2] and [Popov and Vinberg 1994, Theorem 1.5]). Hence we may (and shall) assume that $Z$ is an irreducible smooth affine algebraic variety.

Since $G$ is reductive, $k[Z]^{G}$ is a finitely generated $k$-algebra (see, e.g., [Mumford and Fogarty 1982, Theorem A.1.0] and the references therein). Denote by $Z / / H$ the affine algebraic variety $\operatorname{Specm}\left(k[Z]^{G}\right)$ and by $\pi: Z \rightarrow Z / / H$ the morphism corresponding to the inclusion homomorphism $k[Z]^{G} \hookrightarrow k[Z]$.

The condition on the point $a$ implies that its $H$-stabilizer is trivial,

$$
H_{a}=\{e\} .
$$


Hence $H \cdot a$ is a closed $H$-orbit of maximal dimension. Taking into account that in every fiber of $\pi$ there is a unique closed orbit lying in the closure of every orbit contained in this fiber (see [Mumford and Fogarty 1982, Corollaries 1.2, A.1.0]), from this we deduce the equality

$$
\pi^{-1}(\pi(a))=H \cdot a .
$$

Since the group $\{e\}$ is linearly reductive, from (27) and the separability of $\varphi$, we infer by [Bardsley and Richardson 1985, Proposition 7.6] that there is a smooth affine subvariety $S$ of the $H$-variety $Z$, which is an étale slice at $a \in S$. In view of (27), this means the following:

(i) The morphisms

$$
\left.\pi\right|_{S}: S \rightarrow Z / / H \quad \text { and } \quad \psi: H \times S \rightarrow Z, \quad(h, s) \mapsto h \cdot s
$$

are étale.

(ii) The diagram

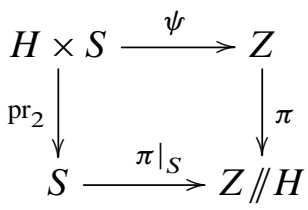

is a Cartesian square; i.e., it is commutative and the morphism

$$
H \times S \rightarrow S \times Z / / H Z
$$

determined by $\psi$ and $\mathrm{pr}_{2}$ is an isomorphism.

From (i) and (ii), we deduce that $\pi(a)$ is a smooth point of $Z / / H$ and the differentials $d_{(e, a)} \psi, d_{a}\left(\left.\pi\right|_{S}\right)$ are isomorphisms. Since $d_{(e, a)} \mathrm{pr}_{2}$ is clearly surjective, (ii) then implies that $d_{a} \pi$ is surjective, too.

Now, in view of (28) and transitivity of the action of $H$ on $H \cdot a$, we conclude that $d_{z} \pi$ is surjective for every point $z \in \pi^{-1}(\pi(a))$. In view of Lemma 8 , this implies the claim of Theorem 7.

Theorem 9. The ideal $\operatorname{ker} \mu$ in $\mathcal{S}^{+} \otimes_{k} \mathcal{S}^{-}$is generated by $(\operatorname{ker} \mu)^{G}$.

Proof. In the proof of Theorem 3, we have shown that

— the homomorphism $\mu$ is the composition of the homomorphisms (17);

- the morphism $\gamma$ is a closed embedding.

In view of these facts, Theorem 9 is equivalent to the claim that the ideal ker $\gamma^{*}$ in $k[X \times X]$ is generated by $\left(\operatorname{ker} \gamma^{*}\right)^{G}$. This claim follows from Theorem 7 . 


\section{Structure of $(\operatorname{ker} \mu)^{G}$}

We shall use the following lemma for describing $(\operatorname{ker} \mu)^{G}$ as a vector space.

\section{Lemma 10.}

$$
\begin{aligned}
\operatorname{dim}\left(\mathcal{S}^{+}(\lambda) \otimes_{k} \mathcal{S}^{-}(v)\right)^{G} & =\left\{\begin{array}{ll}
1 & \text { if } v=\lambda^{*}, \\
0 & \text { if } v \neq \lambda^{*}
\end{array} \quad \text { for every } \lambda, v \in \mathrm{X}(T)_{+},\right. \\
\left(\mathcal{S}^{+} \otimes_{k} \mathcal{S}^{-}\right)^{G} & =\bigoplus_{\lambda \in \mathrm{X}(T)_{+}}\left(\mathcal{S}^{+}(\lambda) \otimes_{k} \mathcal{S}^{-}\left(\lambda^{*}\right)\right)^{G} .
\end{aligned}
$$

Proof. In view of (8), the equality (30) follows from (29). To prove (29), we note that

$$
\left(\mathcal{S}^{+}(\lambda) \otimes_{k} \mathcal{S}^{-}(v)\right)^{G} \cong \operatorname{Hom}_{G}\left(\mathcal{S}^{+}(\lambda)^{*}, \mathcal{S}^{-}(v)\right)
$$

and, in view of (6), the $G$-module $\mathcal{S}^{+}(\lambda)^{*}$ is the universal highest weight module of weight $\lambda$ (the Weyl module); in particular, for each $G$-module $M$, there is an isomorphism

$$
\operatorname{Hom}_{G}\left(\mathcal{S}^{+}(\lambda)^{*}, M\right) \stackrel{\cong}{\longrightarrow}\left(M^{U^{+}}\right)_{\lambda},
$$

where the right-hand side of (31) is the weight space of $T$ (see [Jantzen 1987, II.2.13, Lemma]). Since $\mathcal{S}^{-}(v)^{U^{+}}$is a line on which $B^{+}$acts by means of $v^{*}$ (see [Jantzen 1987, II.2.2, Proposition]), this proves (29).

We identify $k[G] \otimes_{k} k[G]$ with $k[G \times G]$ by the isomorphism

$$
k[G] \otimes_{k} k[G] \rightarrow k[G \times G], \quad f_{1} \otimes f_{2} \mapsto\left((a, b) \mapsto f_{1}(a) f_{2}(b)\right) .
$$

Thus $\mathcal{S}^{+} \otimes_{k} \mathcal{S}^{-}$is regarded as a subalgebra of $k[G \times G]$, and (4), (32) yield the equality

$$
f(a, a)=\mu(f)(a) \text { for every } f \in \mathcal{S}^{+} \otimes_{k} \mathcal{S}^{-} \text {and } a \in G .
$$

Theorem 11. (i) If $f \in\left(\mathcal{S}^{+} \otimes_{k} \mathcal{S}^{-}\right)^{G}$, then $f-f(e, e) \in(\operatorname{ker} \mu)^{G}$.

(ii) Every $h \in(\operatorname{ker} \mu)^{G}$ can be uniquely written in the form

$$
h=\sum\left(h_{\lambda}-h_{\lambda}(e, e)\right), \quad h_{\lambda} \in\left(\mathcal{S}^{+}(\lambda) \otimes_{k} \mathcal{S}^{-}\left(\lambda^{*}\right)\right)^{G},
$$

where the sum is taken over a finite set of nonzero elements $\lambda \in \mathrm{X}(T)_{+}$.

Proof. (i) Since $\mu$ is $G$-equivariant, its restriction to $\left(\mathcal{S}^{+} \otimes_{k} \mathcal{S}^{-}\right)^{G}$ is a homomorphism to $k[G]^{G}=k$. Hence $\mu(f)$ is a constant. In view of (33), this implies (i).

(ii) If (34) holds, then the decomposition (30) implies that $h_{\lambda}$ is the natural projection of $h$ to $\left(\mathcal{S}^{+}(\lambda) \otimes_{k} \mathcal{S}^{-}\left(\lambda^{*}\right)\right)^{G}$ determined by this decomposition, whence the uniqueness of (34). To prove the existence, let $h_{\lambda}$ be the aforementioned projection of $h$ to $\left(\mathcal{S}^{+}(\lambda) \otimes_{k} \mathcal{S}^{-}\left(\lambda^{*}\right)\right)^{G}$. Then $h=\sum_{\lambda \in F} h_{\lambda}$ for a finite set $F \subset \mathrm{X}(T)_{+}$. 
Hence $0=\mu(h)=\sum_{\lambda \in F} \mu\left(h_{\lambda}\right)$. As above, $\mu\left(h_{\lambda}\right)=h_{\lambda}(e, e)$; this implies equality (34), where the sum is taken over all $\lambda \in F$. Since $h_{0}$ is a constant, we may assume that $F$ does not contain 0 . This proves (ii).

In the next lemma, for brevity, we put (cf. [Jantzen 1987])

$$
\begin{gathered}
V(\lambda):=\mathcal{S}^{-}(\lambda)^{*} \cong \mathcal{S}^{+}(\lambda)^{*}, \quad L(\lambda):=V(\lambda) / \operatorname{rad}_{G} V(\lambda), \\
\pi_{\lambda}: V(\lambda) \rightarrow L(\lambda) \text { is the canonical projection. }
\end{gathered}
$$

The $G$-module $V(\lambda)$ (hence $L(\lambda)$ as well) is generated by a $B^{+}$-stable line of weight $\lambda$ (see [Jantzen 1987, II.2.13, Lemma]), whence $V(\lambda)$ is also generated by a $B^{-}$-stable line of weight $-\lambda^{*}$.

Also, for the $G$-modules $P$ and $Q$, we denote by $\mathscr{B}(P \times Q)$ the $G$-module of all bilinear maps $P \times Q \rightarrow k$; we then have the isomorphism of $G$-modules

$$
P^{*} \otimes_{k} Q^{*} \stackrel{\cong}{\rightrightarrows}(P \times Q), \quad f \otimes h \mapsto f h .
$$

Lemma 12. For all elements $\lambda, v \in \mathrm{X}(T)_{+}$, the following hold:

(a) $\operatorname{dim} \mathscr{B}(V(\lambda) \times V(v))^{G}=\left\{\begin{array}{ll}1 & \text { if } v=\lambda^{*} \\ 0 & \text { if } v \neq \lambda^{*}\end{array}\right.$.

(b) $\operatorname{dim} \mathscr{B}(L(\lambda) \times L(v))^{G}=\left\{\begin{array}{ll}1 & \text { if } v=\lambda^{*} \\ 0 & \text { if } v \neq \lambda^{*}\end{array}\right.$.

(c) Every nonzero element $\theta \in \mathscr{B}\left(L(\lambda) \times L\left(\lambda^{*}\right)\right)^{G}$ is a nondegenerate pairing $L(\lambda) \times L\left(\lambda^{*}\right) \rightarrow k$.

(d) If $l^{+} \in L(\lambda), l^{-} \in L\left(\lambda^{*}\right)$ are the nonzero semi-invariants of, respectively, $B^{+}$and $B^{-}$, then $\theta\left(l^{+}, l^{-}\right) \neq 0$ for $\theta$ from (c). For every nonzero element $\epsilon \in k$, there exists a unique $\theta$ such that $\theta\left(l^{+}, l^{-}\right)=\epsilon$.

(e) Every element $\vartheta \in \mathscr{B}\left(V(\lambda) \times V\left(\lambda^{*}\right)\right)^{G}$ vanishes on $\operatorname{ker} \pi_{\lambda} \times \operatorname{ker} \pi_{\lambda^{*}}$. If $\vartheta \neq 0$, then $\vartheta$ is a nondegenerate pairing $V(\lambda) \times V\left(\lambda^{*}\right) \rightarrow k$.

(f) Let $v^{+} \in V(\lambda)$ and $v^{-} \in V\left(\lambda^{*}\right)$ be, respectively, the nonzero $B^{+}$- and $B^{-}$-semiinvariants of weights $\lambda$ and $-\lambda$ that generate the $G$-modules $V(\lambda)$ and $V\left(\lambda^{*}\right)$. Then $\vartheta\left(v^{+}, v^{-}\right) \neq 0$ for every nonzero element $\vartheta \in \mathscr{B}\left(V(\lambda) \times V\left(\lambda^{*}\right)\right)^{G}$.

Proof. Part (a) follows from (29), (36), (35). Part (b) is proved similarly, using that $L(\lambda)$ is a simple $G$-module with highest weight $\lambda$ (see [Jantzen 1987, II.2.4]). The simplicity of $L(\lambda)$ implies (c) because the left and right kernels of $\theta$ are $G$-stable.

Proving (d), take a basis $\left\{p_{1}, \ldots, p_{s}\right\}$ of $L(\lambda)$ such that $p_{1}=l^{+}$and every $p_{i}$ is a weight vector of $T$. Let $\left\{p_{1}^{*}, \ldots, p_{s}^{*}\right\}$ be the basis of $L\left(\lambda^{*}\right)$ dual to $\left\{p_{1}, \ldots, p_{s}\right\}$ with respect to $\theta$. Let $L(\lambda)^{\prime}$ be the linear span over $k$ of all $p_{i}$ with $i>1$. Then $L(\lambda)^{\prime}$ is $B^{-}$-stable, and, for every element $u \in U^{-}$, we have $u \cdot p_{1}=p_{1}+p^{\prime}$, 
where $p^{\prime} \in L(\lambda)^{\prime}$ (see, e.g., [Steinberg 1974, Section 3.3, Proposition 2 and p. 84]). Then, for all elements $\alpha_{1}, \ldots, \alpha_{s} \in k$, we have

$$
\begin{aligned}
\left(u \cdot p_{1}^{*}\right)\left(\sum_{i=1}^{s} \alpha_{i} p_{i}\right) & =p_{1}^{*}\left(\sum_{i=1}^{s} \alpha_{i}\left(u^{-1} \cdot p_{i}\right)\right) \\
& =p_{1}^{*}\left(\alpha_{1} p_{1}+\text { an element of } L(\lambda)^{\prime}\right) \\
& =\alpha_{1}=p_{1}^{*}\left(\sum_{i=1}^{s} \alpha_{i} p_{i}\right),
\end{aligned}
$$

whence $u \cdot p_{1}^{*}=p_{1}^{*}$. Therefore, $l^{-}=\lambda p_{1}^{*}$ for a nonzero $\lambda \in k$, and hence $\theta\left(l^{+}, l^{-}\right)=\lambda \neq 0$. This and (b) prove $(\mathrm{d})$.

It follows from (35), (a), and (b) that the embedding

$$
\mathscr{B}\left(L(\lambda) \times L\left(\lambda^{*}\right)\right)^{G} \rightarrow \mathscr{B}\left(V(\lambda) \times V\left(\lambda^{*}\right)\right)^{G}, \quad \theta \mapsto \theta \circ\left(\pi_{\lambda} \times \pi_{\lambda^{*}}\right),
$$

is an isomorphism. Part (e) follows from this and (c).

Part (f) follows from (d) and (e), because $\pi_{\lambda}\left(v^{+}\right)$and $\pi_{\lambda^{*}}\left(v^{-}\right)$are, in view of (35), the nonzero semi-invariants of, respectively, $B^{+}$and $B^{-}$.

Lemma 13. Let an algebraic group $H$ act on an algebraic variety $Z$ and let $V$ be a finite-dimensional submodule of the $H$-module $k[Z]$. Then the morphism

$$
\varphi: Z \rightarrow V^{*}, \quad \varphi(a)(f)=f(a) \text { for every } a \in Z, f \in V,
$$

has the following properties:

(i) $\varphi$ is $H$-equivariant.

(ii) The restriction of $\varphi^{*}$ to $\left(V^{*}\right)^{*}$ is an isomorphism $\left(V^{*}\right)^{*} \rightarrow V$.

(iii) $\varphi^{*}$ exercises an isomorphism between $k[\overline{\varphi(Z)}]$ and the subalgebra of $k[Z]$ generated by $V$.

Proof. Part (i) is proved by direct verification.

Every function $f \in V$ determines an element $l_{f} \in\left(V^{*}\right)^{*}$ by the formula $l_{f}(s)=s(f), s \in V^{*}$. It is immediate that $V \rightarrow\left(V^{*}\right)^{*}, f \mapsto l_{f}$ is a vector space isomorphism and that (37) implies $\varphi^{*}\left(l_{f}\right)=f$. This proves (ii).

Let $\iota:\left(V^{*}\right)^{*} \rightarrow k[\overline{\varphi(Z)}]$ be the restriction homomorphism. The $k$-algebra $k[\overline{\varphi(Z)}]$ is generated by $\iota\left(\left(V^{*}\right)^{*}\right)$. Part (iii) now follows from the fact that $\varphi^{*}$ exercises an embedding of $k[\overline{\varphi(Z)}]$ in $k[Z]$ and, in view of (ii), the image of $\iota\left(\left(V^{*}\right)^{*}\right)$ under this embedding is $V$.

Corollary 14. In the notation of Lemma 13 , let $V \neq\{0\}$ and let the orbit $H \cdot a$ be dense in $Z$. Then $\varphi(a) \neq 0$.

We call the morphism (37) the covariant determined by the submodule $V$. 
Lemma 15. Let $\lambda$ be an element of $\mathrm{X}(T)+$ and let

$$
\varphi^{+}: G \rightarrow \mathcal{S}^{+}(\lambda)^{*}, \quad \varphi^{-}: G \rightarrow \mathcal{S}^{-}\left(\lambda^{*}\right)^{*}
$$

be the covariants determined by the submodules $\mathcal{S}^{+}(\lambda)$ and $\mathcal{S}^{-}\left(\lambda^{*}\right)$ of the $G$-module $k[G]$. Then $v^{+}:=\varphi^{+}(e)$ and $v^{-}:=\varphi^{-}(e)$ are, respectively, the nonzero $B^{+}$- and $B^{-}$-semi-invariants of weights $\lambda$ and $-\lambda$.

Proof. First, we have $v^{+} \neq 0, v^{-} \neq 0$ by Corollary 14 . Next, for every $f \in \mathcal{S}^{+}(\lambda)$, $b \in B^{+}$, we have

$$
\begin{aligned}
\left(b \cdot v^{+}\right)(f) & =\varphi^{+}(e)\left(b^{-1} \cdot f\right) \stackrel{(37)}{=}\left(b^{-1} \cdot f\right)(e) \\
& =f(b) \stackrel{(5)}{=} b^{\lambda} f(e) \stackrel{(37)}{=}\left(b^{\lambda} v^{+}\right)(f),
\end{aligned}
$$

whence $b \cdot v^{+}=b^{\lambda} v^{+}$; i.e., $v^{+}$is a nonzero $B^{+}$-semi-invariant of weight $\lambda$, as claimed. For $v^{-}$the proof is similar.

Theorem 16. The restriction of $\mu$ to $\left(\mathcal{S}^{+}(\lambda) \otimes_{k} \mathcal{S}^{-}(\lambda)\right)^{G}$ for every $\lambda \in \mathrm{X}(T)_{+}$is an isomorphism $\left(\mathcal{S}^{+}(\lambda) \otimes_{k} \mathcal{S}^{-}\left(\lambda^{*}\right)\right)^{G \stackrel{\cong}{\longrightarrow}} k[G]^{G}=k$.

Proof. In view of (33) and Lemma 10, the proof is reduced to showing that there is a function $f \in\left(\mathcal{S}^{+}(\lambda) \otimes_{k} \mathcal{S}^{-}\left(\lambda^{*}\right)\right)^{G}$ such that $f(e, e) \neq 0$.

Consider the covariants $\varphi^{+}$and $\varphi^{-}$from Lemma 15 and the $G$-equivariant morphism

$$
\varphi:=\varphi^{+} \times \varphi^{-}: G \times G \rightarrow \mathcal{S}^{+}(\lambda)^{*} \times \mathcal{S}^{-}\left(\lambda^{*}\right)^{*} .
$$

Lemma 12(a) and (35) imply that $\mathscr{B}\left(\mathcal{S}^{+}(\lambda)^{*} \times \mathcal{S}^{-}\left(\lambda^{*}\right)^{*}\right)^{G}$ contains a nonzero element $\vartheta$. By Lemma 13, the function $f:=\vartheta \circ \varphi: G \times G \rightarrow k$ is contained in $\left(\mathcal{S}^{+}(\lambda) \otimes_{k} \mathcal{S}^{-}\left(\lambda^{*}\right)\right)^{G}$. For this $f$, using Lemmas 15 and 12(f), we obtain

$$
f(e, e)=\vartheta(\varphi(e, e))=\vartheta\left(\varphi^{+}(e), \varphi^{-}(e)\right) \neq 0 .
$$

This completes the proof.

Corollary 17. For every element $\lambda \in \mathrm{X}(T)_{+}$, there exists a unique element

$$
s_{\lambda} \in\left(\mathcal{S}^{+}(\lambda) \otimes_{k} \mathcal{S}^{-}\left(\lambda^{*}\right)\right)^{G} \subseteq k[G \times G] \text { such that } s_{\lambda}(e, e)=1 .
$$

If $\left\{f_{1}, \ldots, f_{d}\right\}$ and $\left\{h_{1}, \ldots, h_{d}\right\}$ are the bases of $\mathcal{S}^{+}(\lambda)$ and $\mathcal{S}^{-}\left(\lambda^{*}\right)$ dual with respect to a nondegenerate $G$-invariant pairing $\mathcal{S}^{+}(\lambda) \times \mathcal{S}^{-}\left(\lambda^{*}\right) \rightarrow k$ (the latter exists by (36) and Lemma 12), then $\varepsilon:=\sum_{i=1}^{d} f_{i}(e) h_{i}(e) \neq 0$ and

$$
s_{\lambda}=\varepsilon^{-1}\left(\sum_{i=1}^{d} f_{i} \otimes h_{i}\right) .
$$

Proof. First, note that if $P, Q$ are the finite-dimensional $k G$-modules, $\theta \in \mathscr{B}(P, Q)^{G}$ is a nondegenerate pairing $P \times Q \rightarrow k$, and $\left\{p_{1}, \ldots, p_{m}\right\}$ and $\left\{q_{1}, \ldots, q_{m}\right\}$ are the bases of $P$ and $Q$ dual with respect to $\theta$, then $\sum_{i=1}^{m} p_{i} \otimes q_{i}$ is a nonzero element 
of $\left(P \otimes_{k} Q\right)^{G}$ (not depending on the choice of these bases). Indeed, $\theta$ determines the isomorphism of $G$-modules

$$
\phi: P \otimes_{k} Q \rightarrow \operatorname{Hom}(P, P),
$$$$
(\phi(p \otimes q))\left(p^{\prime}\right)=\theta\left(p^{\prime}, q\right) p, \quad \text { where } p, p^{\prime} \in P, q \in Q .
$$

From (40), we then obtain

$$
\left(\phi\left(\sum_{i=1}^{m} p_{i} \otimes q_{i}\right)\right)\left(p_{j}\right)=\sum_{i=1}^{m} \theta\left(p_{j}, q_{i}\right) p_{i}=\sum_{i=1}^{m} \delta_{i j} p_{i}=p_{j} ;
$$

therefore, $\phi\left(\sum_{i=1}^{m} p_{i} \otimes q_{i}\right)=\mathrm{id}_{P}$, whence the claim.

For $P=\mathcal{S}^{+}(\lambda), Q=\mathcal{S}^{-}\left(\lambda^{*}\right)$, it yields that $\sum_{i=1}^{d} f_{i} \otimes h_{i}$ is a nonzero element of $\left(\mathcal{S}^{+}(\lambda) \otimes_{k} \mathcal{S}^{-}\left(\lambda^{*}\right)\right)^{G}$. Theorem 16 and (33) then complete the proof.

Remark 18. For char $k=0$, there is another characterization of $s_{\lambda}$. Namely, let $u$ be the universal enveloping algebra of Lie $G$. Every $\mathcal{S}^{ \pm}(\lambda)$ is endowed with the natural $U$-module structure. Let $\left\{x_{1}, \ldots, x_{n}\right\}$ and $\left\{x_{1}^{*}, \ldots, x_{n}^{*}\right\}$ be the bases of Lie $G$ dual with respect to the Killing form $\Phi$. Identify Lie $T$ with its dual space by means of $\Phi$. Let $\sigma$ be the sum of all positive roots. For every $\lambda \in \mathrm{X}(T)_{+}$, put

$$
c_{\lambda}:=\Phi(\lambda+\sigma, \lambda)+\Phi\left(\lambda^{*}+\sigma, \lambda^{*}\right)
$$

and consider on the space $\mathcal{S}^{+}(\lambda) \otimes_{k} \mathcal{S}^{-}\left(\lambda^{*}\right)$ the linear operator

$$
\Delta:=\sum_{i=1}^{n}\left(x_{i} \otimes x_{i}^{*}+x_{i}^{*} \otimes x_{i}\right) .
$$

Proposition 19. The following properties of an element $t \in \mathcal{S}^{+}(\lambda) \otimes_{k} \mathcal{S}^{-}\left(\lambda^{*}\right)$ are equivalent:

(i) $t=s_{\lambda}$.

(ii) $\Delta(t)=-c_{\lambda} t$ and $t(e, e)=1$.

Proof. By [Bourbaki 1975, Chapitre VIII, §6.4, Corollaire], the Casimir element $\Omega:=\sum_{i=1}^{n} x_{i} x_{i}^{*} \in U$ acts on any simple $U$-module with the highest weight $\gamma$ as scalar multiplication by $\Phi(\gamma+\sigma, \gamma)$. Since $\Phi(\gamma+\sigma, \gamma)>0$ if $\gamma \neq 0$, the kernel of $\Omega$ in any finite-dimensional $U$-module $V$ coincides with $V^{G}$. We apply this to $V=\mathcal{S}^{+}(\lambda) \otimes_{k} \mathcal{S}^{-}\left(\lambda^{*}\right)$. For any elements $f \in \mathcal{S}^{+}(\lambda), h \in \mathcal{S}^{-}\left(\lambda^{*}\right)$, we deduce from (41), (42) the following:

$$
\begin{aligned}
\Omega(f \otimes h) & =\sum_{i=1}^{n}\left(x_{i} x_{i}^{*}(f) \otimes h+x_{i}^{*}(f) \otimes x_{i}(h)+x_{i}(f) \otimes x_{i}^{*}(h)+f \otimes x_{i} x_{i}^{*}(h)\right) \\
& =\Omega(f) \otimes h+f \otimes \Omega(h)+\Delta(f \otimes h)=c_{\lambda}(f \otimes h)+\Delta(f \otimes h) .
\end{aligned}
$$

Now Corollary 17 and the aforesaid about $\operatorname{ker} \Omega$ complete the proof. 
Theorem 20. Let $\lambda_{1}, \ldots, \lambda_{m}$ be a system of generators of the monoid $\mathrm{X}(T)_{+}$. Then $(\operatorname{ker} \mu)^{G}$ is the linear span over $k$ of all monomials of the form

$$
\left(s_{\lambda_{1}}-1\right)^{d_{1}} \cdots\left(s_{\lambda_{m}}-1\right)^{d_{m}}, \quad \text { where } d_{i} \in \mathbb{N}, d_{1}+\cdots+d_{m}>0,
$$

where $s_{\lambda_{i}}$ is defined in Corollary 17.

Proof. By Theorem 11(i), the linear span $L$ referred to in Theorem 20 is contained in $(\operatorname{ker} \mu)^{G}$. In view of Theorem 11, to prove the converse inclusion $(\operatorname{ker} \mu)^{G} \subseteq L$, we have to show that, for every function

$$
f \in\left(\mathcal{S}^{+}(\lambda) \otimes_{k} \mathcal{S}^{-}\left(\lambda^{*}\right)\right)^{G},
$$

we have $f-f(e, e) \in L$. Since $\lambda_{1}, \ldots, \lambda_{m}$ is a system of generators of $\mathrm{X}(T)_{+}$, there are integers $d_{1}, \ldots, d_{m} \in \mathbb{N}$ such that $\lambda=\sum_{i=1}^{m} d_{i} \lambda_{i}$. From (39) and (8) we then infer that $h:=\prod_{i=1}^{m} s_{\lambda_{i}}^{d_{i}} \in\left(\mathcal{S}^{+}(\lambda) \otimes_{k} \mathcal{S}^{-}\left(\lambda^{*}\right)\right)^{G}$ and $h(e, e)=1$. This, (43), and (29) imply that $f=f(e, e) h$. Therefore,

$$
f-f(e, e)=f(e, e)(h-1)=f(e, e)\left(\prod_{i=1}^{m}\left(\left(s_{\lambda_{i}}-1\right)+1\right)^{d_{i}}-1\right) .
$$

The right-hand side of (44) clearly lies in $L$. This completes the proof.

Theorem 21. Let $\lambda_{1}, \ldots, \lambda_{m}$ be a system of generators of the monoid $\mathrm{X}(T)_{+}$. Then the ideal ker $\mu$ in $\mathcal{S}^{+} \otimes_{k} \mathcal{S}^{-}$is generated by $s_{\lambda_{1}}-1, \ldots, s_{\lambda_{m}}-1$, where $s_{\lambda_{i}}$ is defined in Corollary 17.

Proof. This follows from Theorems 9 and 20.

\section{Presentation of $\mathcal{S}^{ \pm}$}

If the group $G$ is semisimple, then the semigroup $\mathrm{X}(T)_{+}$has no units other than 0 . Hence the set $\mathscr{H}$ of all indecomposable elements of $\mathrm{X}(T)_{+}$is finite,

$$
\mathscr{H}=\left\{\lambda_{1}, \ldots, \lambda_{d}\right\}
$$

generates $\mathrm{X}(T)_{+}$, and every generating set of $\mathrm{X}(T)_{+}$contains $\mathscr{H}$ (see, e.g., [Lorenz 2005, Lemma 3.4.3]). Note that $\mathscr{H}$, called the Hilbert basis of $\mathrm{X}(T)_{+}$, in general is not a free generating system of $\mathrm{X}(T)_{+}$(i.e., it is not true that every element $\alpha \in \mathrm{X}(T)_{+}$may be uniquely expressed in the form $\left.\alpha=\sum_{i=1}^{d} c_{i} \lambda_{i}, c_{i} \in \mathbb{N}\right)$. Namely, it is free if and only if $G=G_{1} \times \cdots \times G_{s}$, where every $G_{i}$ is either a simply connected simple algebraic group or isomorphic to $\mathrm{SO}_{n_{i}}$ for an odd $n_{i}$ (see [Steinberg 1975, §3], [Richardson 1979, Proposition 4.1], [Richardson 1982, Proposition 13.3] and [Popov 2011, Remark 3.16]). In particular, if $G$ is simply connected, then $\mathscr{H}$ coincides with the set of all fundamental weights and generates $\mathrm{X}(T)_{+}$freely. Note that $\lambda_{i}^{*} \in \mathscr{H}$ for every $i$. 
To understand the presentation of $\mathcal{S}^{ \pm}$, denote respectively by $\operatorname{Sym} \mathcal{S}^{ \pm}\left(\lambda_{i}\right)$ and $\operatorname{Sym}^{m} \mathcal{S}^{ \pm}\left(\lambda_{i}\right)$ the symmetric algebra and the $m$-th symmetric power of $\mathcal{S}^{ \pm}\left(\lambda_{i}\right)$. The naturally $\mathbb{N}^{d}$-graded free commutative $k$-algebra

$$
\mathcal{F}^{ \pm}:=\operatorname{Sym}^{ \pm}\left(\lambda_{1}\right) \otimes_{k} \cdots \otimes_{k} \operatorname{Sym}^{ \pm}\left(\lambda_{d}\right)
$$

may be viewed as the algebra of regular functions $k\left[L^{ \pm}\right]$on the vector space

$$
L^{ \pm}:=\mathcal{S}^{ \pm}\left(\lambda_{1}\right)^{*} \oplus \cdots \oplus \mathcal{S}^{ \pm}\left(\lambda_{d}\right)^{*} .
$$

Let $e_{i}$ be the $i$-th unit vector of $\mathbb{N}^{d}$ and let $\mathcal{F}_{p, q}^{ \pm}$be the homogeneous component of $\mathcal{F}^{ \pm}$of degree $e_{p}+e_{q}$. We have the natural isomorphisms of $G$-modules

$$
\varphi_{p, q}^{ \pm}: \mathcal{F}_{p, q}^{ \pm} \stackrel{\cong}{\longrightarrow} \mathcal{S}_{p, q}^{ \pm}:= \begin{cases}\mathcal{S}^{ \pm}\left(\lambda_{p}\right) \otimes_{k} \mathcal{S}^{ \pm}\left(\lambda_{q}\right) & \text { if } p \neq q \\ \operatorname{Sym}^{2} \mathcal{S}^{ \pm}\left(\lambda_{p}\right) & \text { if } p=q .\end{cases}
$$

By Theorems 1 and 2, the natural multiplication homomorphisms

$$
\phi^{ \pm}: \mathcal{F}^{ \pm} \rightarrow \mathcal{S}^{ \pm} \quad \text { and } \quad \psi_{p, q}^{ \pm}: \mathcal{S}_{p, q}^{ \pm} \rightarrow \mathcal{S}^{ \pm}\left(\lambda_{p}+\lambda_{q}\right)
$$

are surjective. Since $\mathcal{F}^{ \pm}$is a polynomial algebra, the surjectivity of $\phi^{ \pm}$reduces finding a presentation of $\mathcal{S}^{ \pm}$by generators and relations to describing $\operatorname{ker} \phi^{ \pm}$. If $d=\operatorname{dim} T$, the following explicit description of $\operatorname{ker} \phi^{ \pm}$is available:

Theorem 22. Let $G$ be a connected semisimple group such that the Hilbert basis (45) freely generates the semigroup $\mathrm{X}(T)_{+}$. Then

(i) the ideal $\operatorname{ker} \phi^{ \pm}$of the $\mathbb{N}^{d}$-graded $k$-algebra $\mathcal{F}^{ \pm}$is homogeneous;

(ii) this ideal is generated by the union of all its homogeneous components of the total degree 2;

(iii) the set of these homogeneous components coincides with the set of all subspaces $\left(\varphi_{p, q}^{ \pm}\right)^{-1}\left(\operatorname{ker} \psi_{p, q}^{ \pm}\right), 1 \leqslant p \leqslant q \leqslant d$.

Proof. This is the main result of [Kempf and Ramanathan 1987].

Remark 23. In characteristic 0 , for the first time the proof of Theorem 22 was obtained (but not published) by B. Kostant; his proof appeared in [Lancaster and Towber 1979, Theorem 1.1]. In this case, (47) and the surjectivity of $\psi_{p, q}^{ \pm}$yield that $\psi_{p, q}^{ \pm}$is the projection of $\mathcal{S}_{p, q}^{ \pm}$to the Cartan component of $\mathcal{S}_{p, q}^{ \pm}$, and $\operatorname{ker} \psi_{p, q}^{ \pm}$is the unique $G$-stable direct complement to this component. The subspace $\operatorname{ker} \psi_{p, q}^{ \pm}$ admits the following description using the notation of Remark 18 [loc. cit.]. Let $\left\{x_{1}, \ldots, x_{n}\right\}$ and $\left\{x_{1}^{*}, \ldots, x_{n}^{*}\right\}$ be the dual bases of Lie $G$ with respect to $\Phi$. Then $\operatorname{ker} \psi_{p, q}^{+}$is the image of the linear transformation $\left(\sum_{s=1}^{n}\left(x_{s} \otimes x_{s}^{*}+x_{s}^{*} \otimes x_{s}\right)\right)-$ $2 \Phi\left(\lambda_{p}^{*}, \lambda_{q}^{*}\right)$ id of the vector space $\mathcal{S}_{p, q}^{ \pm}$. 
Summing up, if $G$ is a connected semisimple group such that the Hilbert basis (45) freely generates the semigroup $\mathrm{X}(T)_{+}$, then the sought-for canonical presentation of $k[G]$ is given by the surjective homomorphism

$$
\phi:=\phi^{+} \otimes \phi^{-}: \mathcal{F}:=\mathcal{F}^{+} \otimes_{k} \mathcal{F}^{-} \rightarrow k[G]
$$

of the polynomial $k$-algebra $\mathcal{F}$ and the following generating system $\mathscr{R}$ of the ideal ker $\phi$. Identify $\mathcal{F}^{+}$and $\mathcal{F}^{-}$with subalgebras of $\mathcal{F}$ in the natural way. Then $\mathscr{R}=\mathscr{R}_{1} \sqcup \mathscr{R}_{2}$, where

$$
\mathscr{R}_{1}=\bigcup_{p, q}\left(\left(\varphi_{p, q}^{+}\right)^{-1}\left(\operatorname{ker} \psi_{p, q}^{+}\right) \cup\left(\varphi_{p, q}^{-}\right)^{-1}\left(\operatorname{ker} \psi_{p, q}^{-}\right)\right)
$$

(see the definition of $\varphi_{p, q}^{ \pm}, \psi_{p, q}^{ \pm}$in (47), (48)) and

$$
\mathscr{R}_{2}=\left\{s_{\lambda_{1}}-1, \ldots, s_{\lambda_{d}}-1\right\}
$$

(see the definition of $s_{\lambda_{i}}$ in Corollary 17). The elements of $\mathscr{R}_{1}$ (respectively, $\mathscr{R}_{2}$ ) are the Plücker-type (respectively, the $\mathrm{SL}_{2}$-type) relations of the presentation.

The canonical presentation of $k[G]$ is redundant. To reduce the size of $\mathscr{R}_{1}$, we may replace every space $\operatorname{ker} \psi_{p, q}^{ \pm}$in (50) by a basis of this space. Finding such a basis falls within the framework of Standard Monomial Theory.

\section{An example}

As an illustration, here we explicitly describe the canonical presentation of $k[G]$ for $G=\mathrm{SL}_{n}, n \geqslant 2$, and char $k=0$.

Let $T$ be the maximal torus of diagonal matrices in $G$, and let $B^{+}$(respectively, $B^{-}$) be the Borel subgroup of lower (respectively, upper) triangular matrices in $G$. Then

$$
\begin{gathered}
\mathscr{H}=\left\{\varpi_{1}, \ldots, \varpi_{n-1}\right\}, \quad \text { where } \\
\varpi_{d}: T \rightarrow k, \quad \operatorname{diag}\left(a_{1}, \ldots, a_{n}\right) \mapsto a_{n-d+1} \cdots a_{n} .
\end{gathered}
$$

Every pair $i_{1}, i_{2} \in[n]$ determines the function

$$
x_{i_{1}, i_{2}}: G \rightarrow k, \quad\left(\begin{array}{ccc}
a_{1,1} & \ldots & a_{1, n} \\
\ldots & \ldots & \ldots \\
a_{n, 1} & \ldots & a_{n, n}
\end{array}\right) \mapsto a_{i_{1}, i_{2}} .
$$

The $k$-algebra generated by all functions $(52)$ is $k[G]$.

For every $d \in[n-1]$ and every sequence $i_{1}, \ldots, i_{d}$ of $d$ elements of $[n]$, put

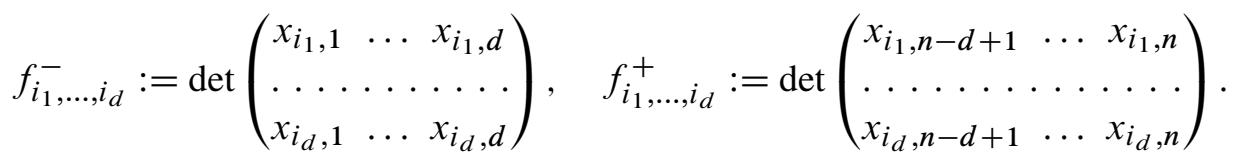


For every fixed $d$, all functions $f_{i_{1}, \ldots, i_{d}}^{-}$(respectively, $f_{i_{1}, \ldots, i_{d}}^{+}$) such that $i_{1}<\cdots<i_{d}$ are linearly independent over $k$ and their linear span over $k$ is the simple $G$-module $\mathcal{S}^{-}\left(\varpi_{d}\right)$ (respectively, $\mathcal{S}^{+}\left(\varpi_{d}\right)$ ); see, e.g., [Flath and Towber 1992, Proposition 3.2]. Therefore, denoting by $x_{i_{1}, \ldots, i_{d}}^{ \pm}$the element $f_{i_{1}, \ldots, i_{d}}^{ \pm}$of the $k$-algebra $\mathcal{F}^{ \pm}$defined by (46), we identify $\mathcal{F}^{ \pm}$with the polynomial $k$-algebra in variables $x_{i_{1}, \ldots, i_{d}}^{ \pm}$, where $d$ runs over $[n-1]$ and $i_{1}, \ldots, i_{d}$ runs over $[n]_{d}$. Correspondingly, the $k$-algebra $\mathcal{F}$ is identified with the polynomial $k$-algebra in the variables $x_{i_{1}, \ldots, i_{d}}^{-}$and $x_{i_{1}, \ldots, i_{d}}^{+}$, the homomorphism (49) takes the form

$$
\phi: \mathcal{F} \rightarrow k[G], \quad x_{i_{1}, \ldots, i_{d}}^{+} \mapsto f_{i_{1}, \ldots, i_{d}}^{+}, \quad x_{i_{1}, \ldots, i_{d}}^{-} \mapsto f_{i_{1}, \ldots, i_{d}}^{-},
$$

and $\phi^{ \pm}=\left.\phi\right|_{\mathcal{F}^{ \pm}}$. Below the sets (50) and (51) are explicitly specified using this notation.

First, we will specify the Plücker-type relations. It is convenient to introduce the following elements of $\mathcal{F}^{ \pm}$. Let $i_{1}, \ldots, i_{d}$ be a sequence of $d \in[n-1]$ elements of $[n]$, and let $j_{1}, \ldots, j_{d}$ be the nondecreasing sequence obtained from $i_{1}, \ldots, i_{d}$ by permutation. Then we put

$$
x_{i_{1}, \ldots, i_{d}}^{ \pm}= \begin{cases}\operatorname{sgn}\left(i_{1}, \ldots, i_{d}\right) x_{j_{1}, \ldots, j_{d}}^{ \pm} & \text {if } i_{p} \neq i_{q} \text { for all } p \neq q \\ 0 & \text { otherwise }\end{cases}
$$

The $k$-algebra $\mathcal{S}^{ \pm}$is the coordinate algebra of the affine multicone over the flag variety; see [Towber 1979]. By the well-known classical Hodge's result [1942; 1943] (see also [Towber 1979, p. 434, Corollary 1]), the ideal $\operatorname{ker} \phi^{ \pm}$is generated by all elements of the form

$$
\sum_{l=1}^{q+1}(-1)^{l} x_{i_{1}, \ldots, i_{p-1}, j_{l}}^{ \pm} x_{j_{1}, \ldots, \hat{j}_{l}, \ldots, j_{q+1}}^{ \pm},
$$

where $p$ and $q$ run over $[n-1], p \leqslant q$, and $i_{1}, \ldots, i_{p-1}$ and $j_{1}, \ldots, j_{q+1}$ run over $[n]_{p-1}$ and $[n]_{q+1}$ respectively. Since every element (53) is homogeneous of degree 2, this result together with Theorem 22 imply that, for every fixed $p, q \in[n-1]$, the set $\left(\varphi_{p, q}^{ \pm}\right)^{-1}\left(\operatorname{ker} \psi_{p, q}^{ \pm}\right)$in (50) is the linear span of all elements (53), where $i_{1}, \ldots, i_{p-1}$ and $j_{1}, \ldots, j_{q+1}$ run over $[n]_{p-1}$ and $[n]_{q+1}$ respectively. This describes the Plücker-type relations (50).

Secondly, we will describe $s_{\varpi_{d}}$. If $\boldsymbol{i} \in[n]_{n-d}$ is a sequence $i_{1}, \ldots, i_{n-d}$, we put $x_{\boldsymbol{i}}^{ \pm}:=x_{i_{1}, \ldots, i_{n-d}}^{ \pm}$and denote by $\boldsymbol{i}^{*} \in[n]_{d}$ the unique sequence $j_{1}, \ldots, j_{d}$ whose intersection with $i_{1}, \ldots, i_{n-d}$ is empty. Let $\operatorname{sgn}\left(\boldsymbol{i}, \boldsymbol{i}^{*}\right)$ be the sign of the permutation $\left(i_{1}, \ldots, i_{n-d}, j_{1}, \ldots, j_{d}\right)$. Then by [Flath and Towber 1992, Theorem 3.1(b)],

$$
s_{\varpi_{d}}=\sum_{\boldsymbol{i} \in[n]_{n-d}} \operatorname{sgn}\left(\boldsymbol{i}, \boldsymbol{i}^{*}\right) x_{\boldsymbol{i}}^{-} x_{\boldsymbol{i}^{*}}^{+} .
$$

This describes the $\mathrm{SL}_{2}$-type relations (51). 
A similar description of the presentation of $k[G]$ may be given for the classical groups $G$ of several other types: for them, the Plücker-type (respectively, the $\mathrm{SL}_{2}$ type) relations are obtained using [Lancaster and Towber 1979; 1985] (respectively, [Flath and Towber 1992]).

\section{Acknowledgement}

I am grateful to the referee for comments.

\section{References}

[Bardsley and Richardson 1985] P. Bardsley and R. W. Richardson, "Étale slices for algebraic transformation groups in characteristic p", Proc. London Math. Soc. (3) 51:2 (1985), 295-317. MR 86m:14034 Zbl 0604.14037

[Borel 1991] A. Borel, Linear algebraic groups, 2nd ed., Graduate Texts in Mathematics 126, Springer, New York, 1991. MR 92d:20001 Zbl 0726.20030

[Bourbaki 1975] N. Bourbaki, Éléments de mathématique, Fasc. XXXVIII: Groupes et algèbres de Lie. Chapitre VII: Sous-algèbres de Cartan, éléments réguliers. Chapitre VIII: Algèbres de Lie semi-simples déployées, Actualités Scientifiques et Industrielles 1364, Hermann, Paris, 1975. MR 56 \#12077 Zbl 0329.17002

[Flath 1994] D. E. Flath, "Coherent tensor operators", pp. 75-84 in Lie algebras, cohomology, and new applications to quantum mechanics (Springfield, MO, 1992), edited by N. Kamran and P. J. Olver, Contemporary Mathematics 160, Amer. Math. Soc., Providence, RI, 1994. MR 95c:20058 Zbl 0820.22008

[Flath and Towber 1992] D. E. Flath and J. Towber, "Generators and relations for the affine rings of the classical groups", Comm. Algebra 20:10 (1992), 2877-2902. MR 93j:20094 Zbl 0773.20014

[Grothendieck 1958] A. Grothendieck, "Torsion homologique et sections rationnelles", in Anneaux de Chow et applications, Séminaire Claude Chevalley 3, Exposé 5, École Normale Supérieure, Paris, 1958.

[Hodge 1942] W. V. D. Hodge, "A note on k-connexes", Proc. Cambridge Philos. Soc. 38 (1942), 129-143. MR 3,305a JFM 68.0402.02

[Hodge 1943] W. V. D. Hodge, "Some enumerative results in the theory of forms", Proc. Cambridge Philos. Soc. 39 (1943), 22-30. MR 4,184e Zbl 0060.04107

[Jantzen 1987] J. C. Jantzen, Representations of algebraic groups, Pure and Applied Mathematics 131, Academic Press, Boston, 1987. MR 89c:20001 Zbl 0654.20039

[Kempf 1989] G. R. Kempf, "Projective coordinate rings of abelian varieties", pp. 225-235 in Algebraic analysis, geometry, and number theory (Baltimore, MD, 1988), edited by J. I. Igusa, Johns Hopkins University Press, Baltimore, MD, 1989. MR 98m:14047 Zbl 0785.14025

[Kempf and Ramanathan 1987] G. R. Kempf and A. Ramanathan, "Multi-cones over Schubert varieties”, Invent. Math. 87:2 (1987), 353-363. MR 88c:14067 Zbl 0615.14028

[Lancaster and Towber 1979] G. Lancaster and J. Towber, "Representation-functors and flag-algebras for the classical groups I", J. Algebra 59:1 (1979), 16-38. MR 80i:14020 Zbl 0441.14013

[Lancaster and Towber 1985] G. Lancaster and J. Towber, "Representation-functors and flag-algebras for the classical groups, II”, J. Algebra 94:2 (1985), 265-316. MR 86j:20037 Zbl 0616.14038 
[Lange and Birkenhake 1992] H. Lange and C. Birkenhake, Complex abelian varieties, Grundlehren der Mathematischen Wissenschaften 302, Springer, Berlin, 1992. MR 94j:14001 Zbl 0779.14012

[Lorenz 2005] M. Lorenz, Multiplicative invariant theory, Encyclopaedia of Mathematical Sciences 135, Springer, Berlin, 2005. MR 2005m:13012 Zbl 1078.13003

[Mumford 1966] D. Mumford, "On the equations defining abelian varieties. I", Invent. Math. 1 (1966), 287-354. MR 34 \#4269 Zbl 0219.14024

[Mumford and Fogarty 1982] D. Mumford and J. Fogarty, Geometric invariant theory, 2nd ed., Ergebnisse der Mathematik und ihrer Grenzgebiete 34, Springer, Berlin, 1982. MR 86a:14006 Zbl 0504.14008

[Popov 1989] V. L. Popov, "Closed orbits of Borel subgroups", Math. USSR Sbornik 63:2 (1989), 375-392. MR 89c:14073 Zbl 0713.20036

[Popov 1995] V. L. Popov, "Generators and relations of the affine coordinate rings of connected semisimple algebraic groups", preprint, 1995. Presented at the AMS Central Sectional Meeting \#900, (Chicago, 1995).

[Popov 2000] V. L. Popov, "Generators and relations of the affine coordinate rings of connected semisimple algebraic groups", ESI preprint 972, The Erwin Schrödinger International Institute for Mathematical Physics, Vienna, 2000.

[Popov 2011] V. L. Popov, "Cross-sections, quotients, and representation rings of semisimple algebraic groups", Transform. Groups 16:3 (2011), 827-856. MR 2012j:20146 Zbl 1238.20063

[Popov and Vinberg 1994] V. L. Popov and E. B. Vinberg, "Invariant theory", pp. 123-278 in Algebraic geometry IV, edited by A. N. Parshin and I. R. Shafarevich, Encyclopaedia of Mathematical Sciences 55, Springer, Berlin, 1994. MR 92d:14010 Zbl 0789.14008

[Ramanan and Ramanathan 1985] S. Ramanan and A. Ramanathan, "Projective normality of flag varieties and Schubert varieties", Invent. Math. 79:2 (1985), 217-224. MR 86j:14051 Zbl 0553.14023

[Richardson 1979] R. W. Richardson, "The conjugating representation of a semisimple group", Invent. Math. 54:3 (1979), 229-245. MR 81a:14023 Zbl 0424.20035

[Richardson 1982] R. W. Richardson, "Orbits, invariants, and representations associated to involutions of reductive groups”, Invent. Math. 66:2 (1982), 287-312. MR 83i:14042 Zbl 0508.20021

[Rosenlicht 1956] M. Rosenlicht, "Some basic theorems on algebraic groups", Amer. J. Math. 78 (1956), 401-443. MR 18,514a Zbl 0073.37601

[Rosenlicht 1961] M. Rosenlicht, "On quotient varieties and the affine embedding of certain homogeneous spaces”, Trans. Amer. Math. Soc. 101 (1961), 211-223. MR 24 \#A732 Zbl 0111.17902

[Serre 1955] J.-P. Serre, "Faisceaux algébriques cohérents", Ann. of Math. (2) 61 (1955), 197-278. MR 16,953c Zbl 0067.16201

[Shafarevich 2013] I. R. Shafarevich, Basic algebraic geometry, 1: Varieties in projective space, 3rd ed., Springer, Heidelberg, 2013. MR 3100243 Zbl 1273.14004

[Steinberg 1974] R. Steinberg, Conjugacy classes in algebraic groups, Lecture Notes in Mathematics 366, Springer, Berlin, 1974. MR 50 \#4766 Zbl 0281.20037

[Steinberg 1975] R. Steinberg, "On a theorem of Pittie”, Topology 14 (1975), 173-177. MR 51 \#9101 Zbl 0318.22010

[Towber 1979] J. Towber, "Young symmetry, the flag manifold, and representations of GL( $n)$ ", $J$. Algebra 61:2 (1979), 414-462. MR 83d:15022 Zbl 0437.14030

Received May 28, 2015. Revised August 11, 2015. 
VLADIMIR L. POPOV

SteKLOV MATHEMATICAL InSTITUTE

RUSSIAN ACADEMY OF SCIENCES

GUBKINA 8

MosCOW 119991

RUSSIA

and

NATIONAL RESEARCH UNIVERSITY

HIGHER SCHOOL OF ECONOMICS

MYASNITSKAYA 20

Moscow 101000, RUSSIA

popovvl@mi.ras.ru 


\title{
PACIFIC JOURNAL OF MATHEMATICS
}

\author{
msp.org/pjm
}

Founded in 1951 by E. F. Beckenbach (1906-1982) and F. Wolf (1904-1989)

\section{EDITORS}

Don Blasius (Managing Editor)

Department of Mathematics

University of California

Los Angeles, CA 90095-1555

blasius@math.ucla.edu

\author{
Paul Balmer \\ Department of Mathematics \\ University of California \\ Los Angeles, CA 90095-1555 \\ balmer@math.ucla.edu \\ Robert Finn \\ Department of Mathematics \\ Stanford University \\ Stanford, CA 94305-2125 \\ finn@math.stanford.edu \\ Sorin Popa \\ Department of Mathematics \\ University of California \\ Los Angeles, CA 90095-1555 \\ popa@math.ucla.edu
}

\author{
Vyjayanthi Chari \\ Department of Mathematics \\ University of California \\ Riverside, CA 92521-0135 \\ chari@math.ucr.edu \\ Kefeng Liu \\ Department of Mathematics \\ University of California \\ Los Angeles, CA 90095-1555 \\ liu@math.ucla.edu \\ Jie Qing \\ Department of Mathematics \\ University of California \\ Santa Cruz, CA 95064 \\ qing@ cats.ucsc.edu
}

\section{PRODUCTION}

Silvio Levy, Scientific Editor, production@msp.org

\section{SUPPORTING INSTITUTIONS}

ACADEMIA SINICA, TAIPEI

CALIFORNIA INST. OF TECHNOLOGY

INST. DE MATEMÁTICA PURA E APLICADA

KEIO UNIVERSITY

MATH. SCIENCES RESEARCH INSTITUTE

NEW MEXICO STATE UNIV.

OREGON STATE UNIV.

\author{
STANFORD UNIVERSITY \\ UNIV. OF BRITISH COLUMBIA \\ UNIV. OF CALIFORNIA, BERKELEY \\ UNIV. OF CALIFORNIA, DAVIS \\ UNIV. OF CALIFORNIA, LOS ANGELES \\ UNIV. OF CALIFORNIA, RIVERSIDE \\ UNIV. OF CALIFORNIA, SAN DIEGO \\ UNIV. OF CALIF., SANTA BARBARA
}

\author{
Daryl Cooper \\ Department of Mathematics \\ University of California \\ Santa Barbara, CA 93106-3080 \\ cooper@math.ucsb.edu \\ Jiang-Hua Lu \\ Department of Mathematics \\ The University of Hong Kong \\ Pokfulam Rd., Hong Kong \\ jhlu@maths.hku.hk \\ Paul Yang \\ Department of Mathematics \\ Princeton University \\ Princeton NJ 08544-1000 \\ yang@math.princeton.edu
}

These supporting institutions contribute to the cost of publication of this Journal, but they are not owners or publishers and have no responsibility for its contents or policies.

See inside back cover or msp.org/pjm for submission instructions.

The subscription price for 2015 is US \$420/year for the electronic version, and \$570/year for print and electronic.

Subscriptions, requests for back issues and changes of subscribers address should be sent to Pacific Journal of Mathematics, P.O. Box 4163, Berkeley, CA 94704-0163, U.S.A. The Pacific Journal of Mathematics is indexed by Mathematical Reviews, Zentralblatt MATH, PASCAL CNRS Index, Referativnyi Zhurnal, Current Mathematical Publications and Web of Knowledge (Science Citation Index).

The Pacific Journal of Mathematics (ISSN 0030-8730) at the University of California, c/o Department of Mathematics, 798 Evans Hall \#3840, Berkeley, CA 94720-3840, is published twelve times a year. Periodical rate postage paid at Berkeley, CA 94704, and additional mailing offices. POSTMASTER: send address changes to Pacific Journal of Mathematics, P.O. Box 4163, Berkeley, CA 94704-0163.

PJM peer review and production are managed by EditFLOW ${ }^{\circledR}$ from Mathematical Sciences Publishers.

\section{PUBLISHED BY}

\section{mathematical sciences publishers \\ nonprofit scientific publishing}

http://msp.org/

(C) 2015 Mathematical Sciences Publishers 


\title{
PACIFIC JOURNAL OF MATHEMATICS
}

\author{
Volume 279 No. 1-2 December 2015
}

In memoriam: Robert Steinberg

Robert Steinberg (1922-2014): In memoriam V. S. VARADARAJAN

Cellularity of certain quantum endomorphism algebras

HENNING H. ANDERSEN, GUSTAV I. LEHRER and RUIBIN ZHANG

Lower bounds for essential dimensions in characteristic 2 via orthogonal representations ANTONIO BABIC and VLADIMIR CHERNOUSOV

Cocharacter-closure and spherical buildings

Michael Bate, Sebastian Herpel, Benjamin Martin and Gerhard RöHrLe

Embedding functor for classical groups and Brauer-Manin obstruction

Eva Bayer-Fluckiger, Ting-Yu LeE and Raman Parimala

On maximal tori of algebraic groups of type $G_{2}$

Constantin Beli, PhilipPe Gille and Ting-Yu LeE

On extensions of algebraic groups with finite quotient

MICHEL BRION

Essential dimension and error-correcting codes

SHANE CERNELE and ZiNOVy REICHSTEIN

Notes on the structure constants of Hecke algebras of induced representations of finite Chevalley groups

Charles W. CuRTis

Complements on disconnected reductive groups

FRANÇOIS DIGNE and JEAN MICHEL

Extending Hecke endomorphism algebras

Jie Du, Brian J. Parshall and LeOnard L. SCOTT

Products of partial normal subgroups

ELLEN HENKE

Lusztig induction and $\ell$-blocks of finite reductive groups

RADHA KESSAR and GUNTER MALLE

Free resolutions of some Schubert singularities

Manoj Kummini, Venkatramani Lakshmibai, Pramathanath Sastry and C. S. Seshadri

Free resolutions of some Schubert singularities in the Lagrangian Grassmannian

VenKatramani LAKSHMibai and ReUVEN HODGES

Distinguished unipotent elements and multiplicity-free subgroups of simple algebraic groups

Martin W. Liebeck, Gary M. Seitz and Donna M. Testerman

Action of longest element on a Hecke algebra cell module

GEORGE LUSZTIG

Generic stabilisers for actions of reductive groups

BENJAMIN MARTIN

On the equations defining affine algebraic groups

VLADIMIR L. POPOV

Smooth representations and Hecke modules in characteristic $p$

PETER SCHNEIDER

On CRDAHA and finite general linear and unitary groups

BHAMA SRINIVASAN

Weil representations of finite general linear groups and finite special linear groups PHAM HUU TIEP

The pro- $p$ Iwahori Hecke algebra of a reductive $p$-adic group, $\mathrm{V}$ (parabolic induction) MARIE-FRANCE VIGNÉRAS

Acknowledgement 